\title{
GOBERNANZA DE AGUAS SUBTERRÁNEAS, CONFLICTOS SOCIOAMBIENTALES Y ALTERNATIVAS: EXPERIENCIAS DE COSTA RICA
}

\author{
GROUNDWATER GOVERNANCE, SOCIAL CONFLICTS AND \\ ALTERNATIVES: EXPERIENCES FROM COSTA RICA
}

Gabriela Cuadrado Quesada

Recibido: 01/11/2016 Aceptado: 23/01/2017

\begin{abstract}
Resumen
Este artículo explora los retos de la gobernanza de las aguas subterráneas en Costa Rica y cómo estos tienden a ocasionar conflictos socioambientales. Además, ilustra dichos retos a la luz de dos casos de estudio en Costa Rica, uno en Limón y otro en Guanacaste. Asimismo, analiza las principales demandas hechas por las comunidades que sufren conflictos socioambientales y algunas respuestas de las instituciones públicas. Finalmente, discute cuáles factores son necesarios en las comunidades para que estas logren respuestas favorables de las instituciones del Gobierno. Entre los principales factores analizados se encuentran: crisis hídrica, liderazgo comunal, comunidades de pequeña escala y homogéneas, y demandas legales.

Palabras clave: Gobernanza; aguas subterráneas; conflicto socioambiental; participación; sustentabilidad.

Abstract

This article explores the challenges of groundwater governance and how these challenges tend to generate socioenvironmental conflicts. The paper illustrates such challenges with two case studies from Costa Rica, one in Limon and one in Guanacaste. Moreover, it analyses the main demands made by communities suffering socioenvironmental conflicts and some responses from public institutions. Finally, it discusses what factors are needed in the communities so that they can achieve favourable responses from government institutions. Among the main factors that are discussed are: water crisis; communal leadership; small and homogeneous communities; and lawsuits.
\end{abstract}

Keywords: Governance; groundwater; socioenvironmental conflict; participation; sustainability. 


\section{Introducción ${ }^{1}$}

Las aguas subterráneas son un recurso vasto, a menudo incomprendido y, recurrentemente, subestimado. Constituyen alrededor del 95 por ciento de toda el agua dulce disponible para el consumo humano (Morris et al. 1). Aparte de su uso para actividades domésticas, riego, industria y comercio, las aguas subterráneas son esenciales para el ambiente y los ecosistemas (Boulton et al. 103). A pesar de esto, estudios científicos señalan una preocupante degradación de dichos recursos (WWAP 27).

La sobreexplotación y la contaminación están entre las principales amenazas de las aguas subterráneas, pues son resultado de una gobernanza ineficiente (Varady et al., 2013). Una de las principales y más directa consecuencia cuando existe una gobernanza ineficiente es el incremento de los conflictos socioambientales (Coleman, 2012). Tal vez, el ejemplo más conocido de un conflicto socioambiental ligado al acceso al agua fue el ocurrido en Cochabamba, Bolivia, en 1999, cuando el Gobierno, en respuesta a la presión del Banco Mundial y del Banco Interamericano de Desarrollo, entregó en un fideicomiso la administración del acueducto que abastecía de agua potable a la ciudad de Cochabamba, a un consorcio privado llamado "Aguas del Tunari" (cuyo principal accionista era Betchel Corporation) (Morgan 15). Esto condujo a un incremento desmedido en las tarifas de aguas y el consecuente malestar en los pobladores de Cochabamba, quienes se organizaron y llevaron a cabo un sinnúmero de protestas masivas. Como resultado, el Gobierno rescindió el contrato (Kruse 224).

En los últimos años, se ha dado un esfuerzo concertado por dirigir la atención y mejorar aspectos relacionados con la carencia de gobernanza de las aguas subterráneas y otros afines (Varady et al., 2013), con el fin de evitar conflictos socioambientales. Como resultado, actualmente existe un creciente interés de la literatura por examinar cómo puede alcanzarse una efectiva gobernanza de las aguas subterráneas (Wijnen 14), de manera que se prevengan los conflictos socioambientales. A pesar de esto, aún no es claro cómo poner en funcionamiento una gobernanza eficiente de las aguas subterráneas. Sin embargo, es sabida la importancia que tiene contar con políticas y leyes claras, actualizadas y basadas en estudios científicos (por ejemplo, la cantidad de agua disponible) e instituciones públicas consolidadas capaces de hacer cumplir la legislación.

En Costa Rica se carece de financiamiento para realizar estudios hidrogeológicos, lo cual ha sido determinante en los crecientes retos de la gobernanza de las aguas subterráneas (Arias 2009). Por ejemplo, no existe una cuantificación real de las aguas subterráneas, ni un plan integrado de recursos de aguas subterráneas (Astorga 2009). Por eso, la gobernanza de aguas subterráneas no solo es desordenada, sino que también se basa en la demanda, en lugar de la disponibilidad (Arias 2009). Otros autores han concluido que la condición privilegiada de Costa Rica, que le ha permitido contar con abundancia de aguas superficiales y subterráneas, ha contribuido a tener débiles marcos legales e institucionales (Guzmán-Arias y Calvo-Alvarado 2003). 
Este artículo analiza los retos de gobernanza de las aguas subterráneas en Costa Rica a la luz de dos casos de estudio, uno en Limón y otro en Guanacaste. Asimismo, trata de responder a la siguiente pregunta: ¿hasta qué punto pueden evitarse o manejarse los conflictos socioambientales y alcanzarse una efectiva gobernanza de las aguas subterráneas?

Para poder contestar en detalle esta pregunta, es importante definir qué se entiende en este artículo por conflictos socioambientales y por gobernanza de las aguas subterráneas. Definir un conflicto socioambiental resulta muy complejo porque hay una miríada de definiciones. Sin embargo, a grandes rasgos, se pueden entender como situaciones de confrontación de intereses, derechos y perspectivas en relación con el uso y manejo de los recursos naturales (Coleman), incluido por supuesto el agua. Ahora bien, en el caso de definir qué es la gobernanza de las aguas subterráneas, una de las mejores definiciones la plantea como "un trabajo en progreso" (Shah 205). Para este artículo, se entiende como la manera de regular, usar y manejar los recursos hídricos subterráneos. Incluye la promoción de una acción colectiva responsable para garantizar un uso socialmente sustentable y una protección eficaz de los recursos hídricos subterráneos en beneficio de la humanidad y de los ecosistemas dependientes (Foster y Garduño 2013).

Basado en un análisis de literatura, así como en cuarenta entrevistas cualitativas, se obtuvieron diversas perspectivas de los retos que enfrenta la gobernanza de las aguas subterráneas, en particular, y la ambiental, en general. Por ejemplo, la investigación en Guácimo, Limón, revela cómo el pobre actuar de las instituciones gubernamentales, por ejemplo el Ministerio de Agricultura y Ganadería (MAG) al regular actividades agrícolas tales como el cultivo de la piña, desencadena una lucha socioambiental que termina por exigir a la municipalidad aplicar regulaciones locales.

La investigación en Santa Cruz, Guanacaste, evidencia que fue necesario un conflicto socioambiental para detener una concesión de agua a un hotel, pues ponía en riesgo la sustentabilidad de los recursos hídricos para varias comunidades costeras. Asimismo, muestra cómo el conflicto ha ayudado a mejorar la gobernanza de las aguas subterráneas. En la sección de metodología, se explica en detalle la escogencia de los dos casos de estudio.

Pese a que los dos casos ilustran una amplia variedad de temas y problemas, al compararlos y contrastarlos, se encontraron tres situaciones comunes e interconectadas: los conflictos socioambientales se originan en la falta de ejecución de los marcos legales y políticas de las instituciones públicas, el involucramiento de las comunidades en los conflictos socioambientales responde a una sensación de crisis y, por último, el involucramiento de las comunidades en estos conflictos tiende a ser más exitoso cuando hay liderazgo fuerte y se trata de comunidades homogéneas y en pequeña escala.

Dados estos hallazgos, el artículo propone brindar lecciones empíricas para mejorar el ejercicio de la gobernanza de las aguas subterráneas y para evitar futuros conflictos socioambientales. Además, discute lecciones basadas en testimonios de vecinos y vecinas de las comunidades afectadas, organizaciones no gubernamentales (ONG), 
organizaciones comunitarias e instituciones públicas a nivel central y local y, de esta manera, amplía la literatura sobre los temas aquí tratados.

Posterior a esta introducción, el artículo se desarrolla en tres partes. Primero, se hace una descripción general de la metodología, seguida por la sinopsis del marco legal e institucional de las aguas subterráneas en Costa Rica. Posteriormente, se analizan los dos casos de estudio que incluyen el contexto, el papel de las entidades gubernamentales y las respuestas de las comunidades ante emergentes conflictos socioambientales. De igual manera, los dos casos de estudio discuten los problemas y retos para alcanzar una gobernanza eficaz de las aguas subterráneas. Finalmente, se esboza la conclusión del artículo.

\section{Metodología}

Este artículo combina varios métodos, específicamente el empírico y el doctrinal. En primer lugar, se basa en una investigación doctrinal mediante el análisis de los conceptos de gobernanza de aguas subterráneas, conflictos socioambientales y principios de participación, rendición de cuentas y sustentabilidad, como una manera de definir la gobernanza eficiente de las aguas subterráneas. Luego, se analiza el marco legal e institucional de las aguas subterráneas en Costa Rica y, finalmente, se desarrolla un componente empírico mediante 40 entrevistas cualitativas realizadas a expertos en el uso y manejo de las aguas subterráneas, las cuales se analizaron a la luz de la teoría adaptativa. ${ }^{2}$

\section{Análisis doctrinal}

El método de análisis doctrinal empleado en esta investigación se basa en un estudio documental que aborda, primero, los conceptos de gobernanza de las aguas subterráneas y de los conflictos socioambientales. Posteriormente, analiza las nociones de participación, rendición de cuentas y sustentabilidad, los cuales son fundamentales para alcanzar una gobernanza eficiente de las aguas subterráneas. Los documentos revisados incluyen publicaciones académicas e investigaciones científicas realizadas por organizaciones internacionales como el Global Environmental Fund (GEF) y la Organización de las Naciones Unidas para la Educación, la Ciencia y la Cultura (UNESCO). Asimismo, el artículo estudia el contenido de varias leyes ambientales costarricenses, como la Ley de Aguas, la Ley Orgánica del Ambiente y la Ley Forestal, las cuales regulan el uso y el manejo del agua y su marco institucional.

\section{Análisis empírico}

Nada sustituye la realización de casos de estudio en el campo y la evaluación de los resultados (Yin 16); por lo tanto, en este artículo estos evidencian cómo funciona 
la gobernanza de las aguas subterráneas en la práctica y, gracias a esto, permiten moldear o refinar la teoría para que explique mejor la realidad (Zartman 4).

Las principales fuentes de consulta de esta investigación fueron las entrevistas cualitativas realizadas a quienes trabajan directamente en el manejo de las aguas subterráneas en instituciones gubernamentales; por ejemplo, el Servicio Nacional de Riego y Avenamiento (SENARA), el Ministerio de Ambiente y Energía (MINAE), el Ministerio de Agricultura y Ganadería (MAG), el Ministerio de Salud (MinSa), el Instituto Costarricense de Acueductos y Alcantarillados (AyA), y las municipalidades; además, se deben mencionar las organizaciones no gubernamentales (ONG), la empresa privada (hoteles, inmobiliarias y plantaciones piñeras) y las asociaciones administradoras de los acueductos comunales (Asadas). En total, se completaron cuarenta entrevistas (veinte en cada caso de estudio). Para cumplir con responsabilidades éticas y de confidencialidad esta investigación utilizó, al transcribir las entrevistas, un sistema de identificadores. Este sistema consiste en la categorización de cada entrevista utilizando un número de 2 dígitos, seguido de una declaración de la categoría general del participante. El primer dígito se relaciona con el número del caso de estudio de: Guácimo, Limón (1); y comunidades costeras, Santa Cruz (2). El segundo dígito significa el orden en que se realizó la entrevista en cada caso de estudio. Por ejemplo, el identificador de la primera entrevista realizada en Guácimo, Limón sería la entrevista n. 11, ASADAS. 
Tabla 1

TEMAS CENTRALES, SECTORES ENTREVISTADOS, CONTEXTO Y FECHA DE ENTREVISTAS DE LOS CASOS DE ESTUDIO

\begin{tabular}{|c|c|c|}
\hline Casos de estudio & Guácimo, Limón & $\begin{array}{l}\text { Comunidades costeras, } \\
\text { Santa Cruz }\end{array}$ \\
\hline Temas centrales & $\begin{array}{l}\text { Problemas en la calidad de las } \\
\text { aguas subterráneas debido a la } \\
\text { falta de planificación del territorio } \\
\text { y control adecuado de las planta- } \\
\text { ciones agrícolas (principalmente } \\
\text { piña). }\end{array}$ & $\begin{array}{l}\text { Problemas de cantidad de agua sub- } \\
\text { terránea (escasez), falta de planifi- } \\
\text { cación del territorio y control del } \\
\text { desarrollo del turismo y actividades } \\
\text { inmobiliarias. Incluye el análisis de } \\
\text { la gobernanza de las aguas subte- } \\
\text { rráneas a través de Asadas. }\end{array}$ \\
\hline $\begin{array}{l}\text { Sectores entrevista- } \\
\text { dos }\end{array}$ & $\begin{array}{l}20 \text { personas entrevistadas repre- } \\
\text { sentantes de ONG (2), organiza- } \\
\text { ciones comunales (4), plantaciones } \\
\text { piñeras (2), Asadas (4), funciona- } \\
\text { rios y funcionarias del Gobierno a } \\
\text { nivel central (4) y local (4). }\end{array}$ & $\begin{array}{l}20 \text { personas entrevistadas repre- } \\
\text { sentantes de ONG (2), hoteles e } \\
\text { inmobiliarias (2), Asadas (6), orga- } \\
\text { nizaciones comunales (2) y per- } \\
\text { sonal del Gobierno central (4) y } \\
\text { local (4). }\end{array}$ \\
\hline Contexto & $\begin{array}{l}\text { Área rural del Caribe de Costa } \\
\text { Rica, comunidades de pequeña } \\
\text { escala y reconocido apoyo del } \\
\text { gobierno local. }\end{array}$ & $\begin{array}{l}\text { Zona costera rural del Pacífico } \\
\text { Norte de Costa Rica, destino } \\
\text { turístico y de desarrollo inmobi- } \\
\text { liario, comunidades de pequeña } \\
\text { escala con pobre liderazgo del } \\
\text { gobierno local. }\end{array}$ \\
\hline $\begin{array}{l}\text { Período de } \\
\text { entrevistas }\end{array}$ & $\begin{array}{l}\text { La primera ronda fue realizada } \\
\text { en julio del } 2013 \text { y la segunda en } \\
\text { junio del } 2014 .\end{array}$ & $\begin{array}{l}\text { La primera ronda fue realizada } \\
\text { en julio del } 2013 \text { y la segunda en } \\
\text { junio del } 2014 .\end{array}$ \\
\hline
\end{tabular}

Fuente: Elaboración propia.

Este artículo utiliza la teoría adaptativa para analizar la información recopilada en las entrevistas. Dicha teoría facilita que el análisis de las entrevistas con expertos y expertas en el manejo de las aguas subterráneas esté moldeado por la teoría preexistente sobre la gobernanza de las aguas subterráneas, para guiar el estudio de campo. De forma simultánea, permite que los datos recopilados en la formulación y conducción del trabajo empírico sean utilizados para desarrollar y enriquecer la teoría preexistente sobre la gobernanza de las aguas subterráneas (Liamputtong y Douglas 57). 
Esta investigación se enfoca en Costa Rica porque es un país que enfrenta muchos problemas de gobernanza de las aguas subterráneas, especialmente en materia de contaminación y disminución del recurso (Estado de la Nación, 2015, 180). Además, cuenta con un sistema democrático que busca hacer cumplir las leyes y políticas nacionales, incluido el derecho humano de acceso al agua (Ballestero-Salaverry, 2009, 8). ${ }^{3}$

Los dos casos de estudio de esta investigación, específicamente Guácimo de Limón y las comunidades costeras de Santa Cruz, en Guanacaste, fueron seleccionados por ser ejemplos de diferentes problemas (contaminación y disminución del recuso, respectivamente). El caso de las comunidades costeras de Santa Cruz se seleccionó porque es un área con escasa disponibilidad de aguas superficiales, lo cual ha llevado a aumentar el uso de las aguas subterráneas y de problemas relacionados, dado que muchos usuarios compiten por utilizar los recursos en irrigación, ${ }^{4}$ turismo, desarrollo inmobiliario y consumo humano. En cuanto a Guácimo, además de ser un caso de contaminación, también ilustra cómo la municipalidad puso en funcionamiento ciertos controles para solventar problemas relativos a la calidad de aguas subterráneas debido al incremento de plantaciones agrícola principalmente de banano y piña (Ruepert, 2005).

\section{Sinopsis del marco legal e institucional}

Esta sección examina el marco legal e institucional de las aguas superficiales y subterráneas en Costa Rica. Es importante aclarar que, históricamente, el desarrollo de los marcos legales de las aguas, tanto en Costa Rica como en la mayoría de los países del mundo, se ha centrado en las aguas superficiales (por ejemplo, ríos y lagos), dejando rezagada las aguas subterráneas. Esto ha afectado considerablemente la protección de las aguas ubicadas debajo de la superficie.

Una vez mencionado dicho aspecto, vale la pena mencionar que en Costa Rica el marco legal sobre los recursos hídricos es vetusto, fragmentado, débil, confuso e involucra numerosas leyes. Esto es evidente al mencionar que la ley más importante en cuanto a la regulación del agua en Costa Rica es la Ley de Aguas, promulgada en 1942, cuando la realidad del país distaba mucho de la actual. Por ejemplo, en ese entonces la población total era de medio millón de habitantes (hoy son más de 4800 000) (Instituto Nacional de Estadística y Censos). Dicha ley, aunque fue progresista para la época de promulgación; por ejemplo, incluyó por primera vez la figura de la concesión para el uso de las aguas subterráneas (artículo 7, Ley de Aguas) y a pesar de que ha sido objeto de varias reformas, en la actualidad "no responde a la situación social y económica del país y requiere ser revisada y actualizada con urgencia" (De Albuquerque, 2009).

En particular, la ley no incorpora la importancia de principios como la participación, la rendición de cuentas y la sustentabilidad en la gobernanza de las aguas. Es por ello, y con miras a llenar los vacíos legales existentes, que surgieron un sinnúmero 
de leyes y decretos ejecutivos que, de una u otra manera, regulan la gobernanza de las aguas. Algunos de los más importantes se describen detalladamente a continuación.

La Ley Forestal, por ejemplo, es importante para la protección de las aguas, incluidas las subterráneas, debido a que regula las áreas de protección de las nacientes o manantiales. El artículo 33 de dicha ley indica que los manantiales deben protegerse en un radio de cien metros a su alrededor. Esta protección se refiere al establecimiento de límites a la propiedad privada que incluye, por ejemplo, no cortar árboles, no construir y no aplicar sustancias tóxicas como plaguicidas. Adicionalmente, establece que las propiedades adyacentes a los ríos, arroyos y riachuelos deben ser protegidas en, al menos, quince metros a la orilla de su cauce en áreas rurales y diez metros en las urbanas, si se trata de relieve plano, o cincuenta metros, cuando hay pendientes (artículo 33, Ley Forestal). En el caso de los lagos, embalses y áreas de recarga, se deben proteger cincuenta metros a su alrededor.

Por otra parte, la Ley Orgánica del Ambiente establece el carácter de dominio público de las aguas, incluidas las subterráneas y el principio de conservación de dichos recursos (artículo 2.', Ley Orgánica del Ambiente). Además, la ley en mención destaca la responsabilidad del Estado y de las municipalidades de "impulsar la participación activa de los habitantes de Costa Rica en la toma de decisiones relacionadas con la protección del ambiente" (artículo 6.', Ley Orgánica del Ambiente). Otro tema vital abordado en esta ley es que establece los procesos de evaluación de impacto ambiental. Concretamente, el artículo 17 indica que "Las actividades humanas que alteren o destruyan elementos del ambiente ... requerirán una evaluación de impacto ambiental por parte de la Secretaría Técnica Nacional Ambiental (SETENA)[...]. Su aprobación previa... será requisito indispensable para iniciar las actividades, obras o proyectos".

Asimismo, los artículos 22, 23 y 24 de la Ley Orgánica del Ambiente estipulan la necesidad de la participación pública y el acceso a la información de los procesos de evaluación de impacto ambiental. La ley en cuestión también establece, en los artículos 98 y 99, una gama de medidas de protección y sanciones administrativas que pueden aplicar las autoridades estatales cuando se violan disposiciones legales, esto incluye rescindir permisos, suspender actividades y establecer indemnizaciones.

La Ley General de Salud es otro instrumento fundamental para la gobernanza de las aguas subterráneas. Por ejemplo, regula todos los temas relacionados con contaminación. Entre otros, establece la prohibición de contaminar las aguas (artículo 237 de la Ley General de Salud). Esta ley se relaciona con el Código Penal, que establece penas de tres a diez años de prisión para quienes contaminen aguas y pongan en peligro la salud humana (artículo 261, Código Penal).

Complementariamente, la Ley de Biodiversidad indica que el Ministerio de Ambiente es la entidad a cargo y la responsable de hacer políticas y de administrar los recursos hídricos, (artículo 3., Ley de Biodiversidad). Finalmente, la Ley de Conservación de la Vida Silvestre, en su artículo 5., establece el principio de protección de los recursos hídricos. 
En cuanto al marco institucional, la entidad rectora de las aguas en Costa Rica es el Ministerio de Ambiente y Energía (MINAE), que es responsable de definir las políticas y estrategias afines al sector. En el país, las aguas superficiales y las subterráneas son bienes públicos administrados por el Estado y sus instituciones. Esto implica que para usar el agua es necesario contar con un permiso/concesión del Estado. Dentro del MINAE, la Dirección de Aguas es la entidad encargada de otorgar concesiones para el uso de las aguas, tanto superficiales como subterráneas.

Existe, además, una institución clave para la gobernanza de las aguas subterráneas, en particular, que es el Servicio Nacional de Aguas Subterráneas Riego y Avenamiento (SENARA). Dicha entidad es responsable de realizar investigaciones relativas a las aguas subterráneas y realizar el mapeo de los acuíferos. SENARA es una institución autónoma, sujeta a las directrices políticas del Ministerio de Agricultura y Ganadería (MAG) (debido a su enfoque inicial en el riego cuando se crea por primera vez, en 1983). En la actualidad, esta institución tiene un papel vital en el desarrollo de los estudios hidrológicos en algunos de los principales acuíferos de Costa Rica, así como en el diseño de políticas para proteger los recursos hídricos subterráneos, como la Matriz del Agua. ${ }^{5}$ Incluso, la Sala Constitucional ha señalado en varias ocasiones que las opiniones y recomendaciones de SENARA son plenamente vinculantes para todas las instituciones públicas. ${ }^{6}$ El hecho de que SENARA cuente con estas potestades sobre las aguas subterráneas hace que exista una confusión entre las funciones y potestades del MINAE y su Dirección de Aguas y el SENARA. Especialmente cuando estas instituciones presentan estudios técnicos incongruentes.

Por su parte, el Instituto Costarricense de Acueductos y Alcantarillados (AyA) es la institución encargada de normar y garantizar los servicios de agua potable y alcantarillado sanitario. Muy relacionadas con el AyA, están las Asociaciones Administradoras de Sistemas de Acueductos y Alcantarillados Comunales (ASADAS), que merecen atención especial debido a su papel fundamental en el suministro de agua en muchas comunidades en Costa Rica y, también, por su capacidad de gobernanza, de manera que serán el principal tema de análisis en uno de los casos de estudio.

Aproximadamente el 33 por ciento de la población costarricense recibe agua de una ASADA (Dobbin y Sarathy, 2015). Estas organizaciones se amparan en dos cuerpos legales: por un lado, en la Ley de Asociaciones, Ley No. 218 de 1939 y, por otro, en el Reglamento de las Asociaciones Administradoras de Sistemas de Acueductos y Alcantarillados Comunales, Decreto Ejecutivo No. 32529-S-MINAE de 2005. Este decreto ejecutivo establece que el AyA puede delegar la responsabilidad de abastecimiento de agua en la figura de las ASADAS. El AyA, de acuerdo con el artículo 36.7 del Reglamento de las Asociaciones Administradoras de Sistemas de Acueductos y Alcantarillados Comunales, debe capacitar a las ASADAS en temas técnicos, administrativos y legales. No obstante, hasta ahora la formación que ha dado ha sido, en la mayoría de los casos, muy limitada (Dobbin y Sarathy, 2015). Algunos investigadores aseguran 
que el involucramiento de estas organizaciones podría ser mucho más efectivo si se les diera una apropiada capacitación (FANCA, 2006).

En resumen, en Costa Rica el marco legal sobre los recursos hídricos necesita ser urgentemente reformado. Es necesario contar con una ley marco que, por un lado, reconozca las diferencias y retos en el manejo de las aguas subterráneas y, por otro, incluya en un solo cuerpo legal los principios rectores del uso y manejo de los recursos hídricos, los cuales, hasta el momento, han estado regulados en varios cuerpos normativos (como por ejemplo, el uso sustentable y la participación) o son inexistentes (tal es el caso de la rendición de cuentas). En cuanto al marco institucional, es necesario definir con mayor exactitud hasta dónde llegan las potestades de cada institución y establecer mejor coordinación y diálogo entre ellas. Además, particularmente en referencia al marco legal sobre la protección de las aguas subterráneas, la Sala Constitucional ha mencionado que "lamentablemente, se adolece de claridad, precisión y regulaciones completas para la protección de acuíferos, áreas de recarga y zonas de captación de recursos de aguas subterráneas" (Sala Constitucional, 2004-01923).

A continuación, se analizan en detalle los dos casos de estudio objeto de este artículo, Guácimo, Limón y las comunidades costeras de Santa Cruz, Guanacaste.

\section{Análisis de los casos de estudio}

\section{Guácimo, Limón. Contexto}

El cantón de Guácimo se ubica en la provincia de Limón, en el Caribe costarricense. Así como otros cantones de Costa Rica, ${ }^{7}$ en los últimos años Guácimo ha sido afectado por el brusco incremento de plantaciones de piña. Costa Rica se ha convertido en uno de los productores de piña más importantes del mundo y en el primer exportador mundial (Cuadrado-Quesada, 2008). Mientras en los años ochenta se cultivaba alrededor de 5000 hectáreas de piña, en el año 2000 ya había sembradas alrededor de 13000 hectáreas, y para el año 2008 se registraron alrededor de 45000 hectáreas. La piña se exporta principalmente a Europa y a Estados Unidos. La mayoría de las empresas piñeras son subsidiarias de transnacionales como Del Monte y Chiquita (Aguirre y Arboleda, 2008).

El veloz e improvisado incremento de las plantaciones de piña ha generado cuantiosos impactos negativos, tanto ambientales como sociales; impactos que han sido obviados por las instituciones públicas de Costa Rica, en los ámbitos centrales y locales, pues lo común es que se priorice el crecimiento económico. La imagen internacional de conservación del ambiente de que goza Costa Rica no es congruente con la producción de este monocultivo. Entre los problemas principales causados, están el cambio de uso de suelo de grandes territorios que anteriormente eran bosques o se empleaban para otro tipo de cultivos. También hay erosión y contaminación de los 
acuíferos debido al uso intensivo de agroquímicos. Asimismo, las plantaciones de piña se han expandido a las partes altas de las zonas montañosas y han invadido manantiales y áreas de recarga acuífera (Cuadrado-Quesada y Castro-Vargas, 2009).

Las comunidades en el Caribe, históricamente han tenido agua suficiente y de buena calidad para satisfacer sus necesidades. No obstante, dicha situación ha cambiado con el crecimiento acelerado de las plantaciones de piña y con la contaminación que se deriva de dicho crecimiento. El primer estudio que indicó contaminación de las aguas subterráneas en esas comunidades data de 2005 (Ruepert, 2005). Actualmente, las aguas subterráneas de las comunidades de Cairo, Francia y Luisiana, en el cantón de Siquirres, están contaminadas y afectan a miles de personas. Tal como resume un entrevistado:

Ya desde hace unos siete años, el agua para consumo humano en Luisiana, Milano y Cairo ha estado contaminada... esto se debe al uso intensivo de agroquimicos por parte de las compañías piñeras... la población se ha quedado sin acceso a agua limpia, con excepción de la que le brindan los camiones cisternas que envía el gobierno... pero, mucha gente aún ingiere agua contaminada, porque no puede esperar a que pasen los camiones en sus rutas de entrega... y nadie puede evitar que los niños abran los grifos (Asada N. 117,10 de junio, 2014).

Los cantones de Guácimo y Siquirres se encuentran entre los más bajos del Índice de Desarrollo Humano Cantonal de Costa Rica (Programa de las Naciones Unidas para el Desarrollo (PNUD 18). De acuerdo con muchos entrevistados, problemas sociales tales como violencia doméstica y abandono escolar han aumentado de manera considerable desde que las piñeras vinieron a sus comunidades. A pesar de que, al inicio, ellos pensaron que la llegada de las compañías piñeras sería beneficiosa, porque generaría empleo, después de un tiempo concluyeron que ese no era el caso. Como lo resumió un participante: "La pobreza ha empeorado... mucha gente vendido sus tierras a las piñeras... porque quedaron rodeados de piñeras... ya no sembramos lo que comemos... y la mayoría de la población empleada en las plantaciones son de otras comunidades... o inmigrantes nicaragüenses" (Asada N. . 13, 14 de julio, 2013).

\section{El papel de las entidades gubernamentales y la respuesta de las comunidades}

El contexto anterior muestra cómo la producción de la piña no ha estado debidamente regulada. Las entidades gubernamentales, tanto del gobierno central como local que, en principio, se supone son las responsables de controlar y supervisar el desarrollo de las actividades productivas, no han cumplido con sus deberes (CuadradoQuesada, 2014). Fue evidente para las comunidades que existió falta de ejecución del marco legal ambiental por parte de los organismos gubernamentales. Por ejemplo, las comunidades notaron que las plantaciones piñeras se ubicaron en áreas vulnerables, como a la par de los ríos y encima de áreas de recarga acuífera. Por supuesto, 
toda esta situación trajo secuelas nocivas al ambiente y a las comunidades. Una consecuencia directa de esta situación fue el desarrollo de un conflicto socioambiental.

Las comunidades decidieron que si las instituciones del gobierno no cumplen con sus deberes, es necesario involucrarse y presionar para que se tomen acciones. Entre las medidas de presión tomadas por ellas está la organización en grupos, como asociaciones ambientales, las manifestaciones callejeras, el bloqueo de calles y las manifestaciones frente a la municipalidad. Como lo relató una entrevistada: "hubo manifestaciones todas las semanas, los martes, durante todo el 2007... las comunidades estaban muy enojadas... la gente no quería más piñeras... hubo mucha presión para que se controlara la expansión de la piña" (Entidad gubernamental local N. ${ }^{\circ} 11$, 14 de julio, 2013).

Entre las denuncias ambientales que han presentado y ganado las comunidades hubo una que presentó la ASADA La Perla ante la Fiscalía Ambiental Adjunta. Se trató de una denuncia en contra de una plantación piñera ubicada en la comunidad La Perla, que argumentaba que la plantación de piña, entre otras cosas, cambiaba el uso del suelo, generaba erosión, invadía áreas protegidas de los ríos y contaminaba el agua. La denuncia fue ganada en el año 2013; los jueces condenaron a la empresa a "remover el cultivo de piña que invade las áreas de protección los manantiales y de los ríos", que representaban casi la totalidad de la plantación (Resolución Fiscalía Ambiental Adjunta, No. 24536-2013). Varios meses después de que esto ocurriera, la empresa cesó sus funciones. Como indicó un entrevistado: "Para nosotros, esa demanda significa mucho... es una motivación para seguir luchando contra compañías que destruyen el ambiente y violan las leyes costarricenses... ya ellos se fueron" (Organización comunal N.' 12, 14 de julio, 2013).

Otra acción legal interpuesta por estas comunidades fue un procedimiento administrativo presentado por la Asociación pro-Defensa de los Recursos Naturales del Caribe. Fue en contra de una plantación piñera que quería instalarse cerca de la zona de recarga acuífera en la comunidad de Iroquois. El procedimiento administrativo se presentó ante la SETENA que, como se explicó anteriormente (sección 3), revisa las evaluaciones de impacto ambiental de proyectos. Como resultado, la SETENA resolvió no otorgar la viabilidad ambiental al proyecto y la piñera no pudo instalarse allí. Como dijo un participante: "Uno de los logros de nuestra asociación fue detener la instalación de una plantación piñera en la comunidad de Iroquois que sabíamos que iba a invadir el bosque, las áreas protegidas de los manantiales, las áreas de recarga de acuíferos y, eventualmente, contaminaría el agua" (Organización comunal N. 14, 15 de julio, 2013).

La medida más efectiva tomada por esas comunidades fue presionar a la municipalidad. Ellos solicitaron mejor regulación de las plantaciones piñeras y la implementación de leyes ambientales manifestándose frente a la municipalidad y demandando que no querían nuevas plantaciones piñeras en Guácimo. Eso lo hicieron 
semanalmente, durante casi un año (2007). Su protesta fue la razón principal por la cual la municipalidad se interesó por el tema y tomó acciones para dirigir más apropiadamente la gobernanza de los recursos hídricos y así evitar futuros conflictos socioambientales. Así lo explicó una participante: “La municipalidad sintió presión de las comunidades... la gente estaba enojada... sabíamos que, si no hacíamos nada para controlar la expansión de las plantaciones piñeras, enfrentaríamos disturbios civiles... por eso, decidimos poner en funcionamiento una moratoria" (Entidad gubernamental local N. 15,15 de julio, 2013).

El papel de la municipalidad, desde entonces, ha sido positivo y orientado a dirigir de manera más efectiva la gobernanza de las aguas subterráneas y, así, evitar más conflictos socioambientales. Entre las acciones tomadas por la municipalidad debido a la presión comunitaria estuvo la moratoria para controlar la futura expansión de los cultivos de piña en el sur del cantón, donde se ubica la mayoría de las zonas de recarga acuífera. La declaratoria entró en vigencia por primera vez en el 2008 (Concejo Municipal de Guácimo, Acta N. ${ }^{\circ}$ 34-2007).

Dicha herramienta legal ayudó a detener la contaminación de las aguas subterráneas y a proteger puntos esenciales, como zonas de recarga de acuíferos. Esto fue posible porque las plantaciones piñeras no pudieron expandirse hacia el sur del cantón, donde se ubican las áreas de recarga vulnerables.

A pesar de que la moratoria fue una herramienta legal precautoria esencial y ayudó al cantón a proteger sus acuíferos de los impactos negativos de las plantaciones piñeras, ya no está vigente. La Cámara Nacional de Productores y Exportadores de Piña (CANAPEP) presentó con éxito un recurso de amparo contra la municipalidad, bajo el argumento de que violaba el derecho de las personas a la libertad de comercio y a la propiedad privada. La CANAEP ganó el recurso de amparo el 18 de octubre del 2013 (Sala Constitucional de Costa Rica, 2013- 0013939). En esta sentencia, la Sala Constitucional argumentó que:

Se constata una infracción evidente a la motivación razonable de toda actuación administrativa..., los estudios técnicos que dan sustento al acuerdo municipal recurrido, se elaboraron en el 2006 y 2010, con fines y propósitos distintos a los que son objeto de pretensión de este amparo. Tales circunstancias determinan la infracción de los derechos fundamentales..., dado que, no se trata de una motivación suficiente, idónea, necesaria y razonable, pues la suspensión sine die de la actividad empresarial y productiva se sustentó en informes técnicos elaborados años atrás con una finalidad distinta. De otra parte, se trata de una limitación de un derecho fundamental-ejercicio de la libertad de comercio y agro-industria...-impuesta a través de un simple acuerdo municipal que violenta flagrantemente el principio de la reserva de ley en materia de restricciones a los derechos fundamentales (Sala Constitucional de Costa Rica, 2013- 0013939). 
Es importante mencionar que el voto salvado de la sentencia en comentario consideró que:

La impugnación de los acuerdos municipales no es competencia constitucional sino que corresponde alegarse en la vía ordinaria... los gobiernos locales han sido dotados de competencias constitucionales y legales suficientes como para imponer restricciones al ejercicio de ciertos derechos fundamentales como la libertad de empresa y propiedad privada (Sala Constitucional de Costa Rica, 2013- 0013939, voto salvado).

Con el fin de comprender mejor las causas de este conflicto socioambiental, es importante detallar algunos hechos que sucedieron en Guácimo y afectaron a los pobladores y los convencieron de organizarse para presionar a la municipalidad y a otras entidades gubernamentales locales, como el MINAE y el MAG, y presentar acciones legales. Por ejemplo, fue recurrente durante las entrevistas la preocupación de que en Guácimo se sufriera contaminación de agua, como les sucedía a las comunidades vecinas. Tal como declaró una participante:

Un elemento imperativo para que comenzáramos a luchar contra las compañias y las entidades gubernamentales fue que las comunidades cercanas (Cairo, Francia y Luisiana de Siquirres) tenían sus aguas contaminadas debido al uso intensivo de químicos en las plantaciones piñeras... Comprendimos que teníamos que organizarnos para hacer algo y prevenir esa situación aquí (Guácimo) (Organización comunal N. 116,10 de junio, 2014).

Otro aspecto importante hallado en este caso de estudio, el cual ayudó con la organización y la lucha de las comunidades, fue un fuerte liderazgo. Guácimo cuenta con líderes que motivaron a la gente del cantón a involucrarse en la salvaguarda de las aguas subterráneas y la protección de la naturaleza. Estas personas forman parte, entre otros, de asociaciones ambientales, ASADAS y asociaciones de desarrollo comunal. Esto lo deja en evidencia una de las personas entrevistadas:

Las comunidades, aqui en Guácimo, han sido muy fuertes, tenemos buenos líderes... Cuando ellos decían "tenemos que demandar a esta plantación o aquella, porque están violando las leyes ambientales... y van a destruir el bosque y contaminar el agua..." siempre los seguimos... sabíamos que era la única forma de alcanzar resultados (Asada, N. 118, 10 de junio, 2014).

De la mano del liderazgo consolidado, otro elemento que ayudó a la buena organización fue el hecho de que se trataba de comunidades pequeñas y homogéneas. Los habitantes de Guácimo suman alrededor de 40000 y la mayoría de ellos tiene intereses y valores similares. Según las declaraciones de los participantes de esta investigación, para la gente de Guácimo el agua y los recursos naturales son, en definitiva, una prioridad: 
Todos nos conocemos aqui... las comunidades no son muy grandes y pensamos bastante parecido... tenemos valores e intereses similares... queremos que nuestra comunidad continúe teniendo ríos limpios, bellas montañas y muchos animales... creo que eso nos ayudó a pelear contra las piñeras y el gobierno (ASADA N. 119, 11 de junio, 2014).

En resumen, este caso de estudio demuestra algunas de las principales causas de los conflictos ambientales, los motivos de las acciones de la población y cómo lo manejaron las instituciones gubernamentales. Las comunidades de Guácimo aún enfrentan la amenaza de la expansión de las plantaciones agrícolas especialmente las piñeras y todos los problemas que esto conlleva; no obstante, a partir de las situaciones más críticas del conflicto, se han dado algunas mejoras, como un mayor control de la municipalidad. Las comunidades en Guácimo siguen vigilantes y, por ahora, trabajan en nuevas acciones contra varias plantaciones piñeras y entidades gubernamentales.

\section{Comunidades costeras de Santa Cruz, Guanacaste}

\section{Contexto}

Las comunidades costeras del cantón de Santa Cruz se ubican en la provincia deGuanacaste, en el Pacífico Norte de Costa Rica;la cual es una de las zonas más secas del país, pues solo hay tres meses de lluvia al año, lo que causa una pobre distribución de la disponibilidad de las aguas superficiales (SENARA, 2003). Esto ocasiona que la provincia dependa, casi en su totalidad, de las aguas subterráneas (Astorga, 2009).

Para esta investigación, las comunidades costeras de Santa Cruz incluyen Flamingo, Potrero, Brasilito, Lorena, Huacas, Tamarindo y Playa Grande. La mayoría de esta área es un paraíso costero con hermosas playas de arena blanca que son un destino popular entre turistas locales y foráneos. Debido a estas condiciones, la zona ha experimentado un rápido y expansivo crecimiento del turismo y desarrollo inmobiliario durante las últimas décadas.

Estas actividades se han llevado a cabo sin un plan de sustentabilidad que considere la vulnerabilidad de la zona y sus condiciones climáticas. En su lugar, se han orientado al desarrollo de construcciones masivas, las cuales incluyen gran demanda de agua para campos de golf, piscinas, jacuzzis y otros. Este crecimiento acelerado y sin una adecuada planificación evidencia una falta de implementación de leyes ambientales.

Entre los principales problemas ambientales enfrentados en esta zona están: la escasez de agua, intrusión salina, destrucción de manglares, erosión y deforestación. La responsabilidad de la gobernanza de aguas subterráneas en estas comunidades recae, principalmente, en las ASADAS, ya que la mayoría de estas comunidades son abastecidas de agua potable por medio de una ASADA. Un entrevistado lo resume así: 
Aqui en Tamarindo, y en todas las comunidades vecinas, enfrentamos problemas debido al crecimiento inmobiliario y turístico no planificado... principalmente durante la época seca, tenemos escasez de agua... a veces abrimos el grifo y no hay agua... a veces solo tenemos agua temprano en la mañana y muy tarde en la noche (Asada N. . 21, 21 de julio, 2013).

En la actualidad, se discute una alternativa del Gobierno para desarrollar un acueducto regional que solucione, de alguna manera, los problemas de abastecimiento de agua. La propuesta incluye el uso del acuífero de Nimboyores, pero esta es una de las últimas reservas en la zona; a lo cual muchas ASADAS y personas de las comunidades se oponen. Hasta ahora no se ha logrado consenso y hay puntos en conflicto en las posibles soluciones. Tal como lo indicó un participante: "Aquí ha venido la gente de SENARA, del AyA y de la municipalidad de Santa Cruz a decirnos que el acueducto regional va... pero nosotros necesitamos más información... no hemos visto los estudios que demuestren que es sustentable explotar el Nimboyores" (ASADA N. . 214, 20 de junio, 2014).

El caso de estudio de las comunidades costeras de Santa Cruz examina en detalle lo que ha sido llamado por muchos autores (FANCA, 2006) como el mecanismo más participativo en la gobernanza de recursos hídricos en Costa Rica: las ASADAS. La figura de la ASADA ilustra cómo los ciudadanos y ciudadanas han participado en hacer realidad en sus comunidades el derecho humano de acceso al agua.

Esta zona es la segunda más pobre del país y tiene las tasas más altas de pobreza extrema (INEC 2012). Como resultado, los problemas sociales como deserción escolar y consumo de drogas se han incrementado. Por ejemplo, varios de los y las entrevistadas mencionaron que debido a la pobreza sufrida en la zona, cada vez es más común encontrar jóvenes de 13 o 14 años que se ven obligados a dejar sus estudios para aportar económicamente en sus hogares. De acuerdo con lo dicho por muchos participantes, la pobreza en esta zona continúa creciendo, la mayoría de los empleados en el sector turismo ni siquiera son de la zona y cuando reclutan gente de las comunidades, principalmente, es para labores de limpieza y jardinería.

\section{El papel de las ASADAS}

En este caso de estudio, se examina en detalle el papel de las ASADAS. Estas entidades han existido en las comunidades costeras desde hace mucho tiempo. Fueron integradas por vecinos interesados en responder a las necesidades de acceso a agua de las comunidades emergentes, en los años cincuenta. Desde entonces, las ASADAS han estado a cargo del abastecimiento de agua potable. Sin embargo, recientemente, han dado pasos importantes para ir más allá del abastecimiento y ser las principales responsables por la gobernanza del agua. Las ASADAS han sido las líderes principales cuando han estado latentes conflictos socioambientales. 
Estas entidades han demostrado capacidad local para manejar su propio sistema de acueducto, al ofrecer servicios de buena calidad a bajo costo, si se les permite desarrollarse estratégicamente y se les da apoyo gubernamental y entrenamiento adecuado (Monge, Paz y Ovares, 2013). Pero, tal como mostraron las entrevistas realizadas en este caso de estudio, el problema principal que afecta a las ASADAS es la falta de apoyo institucional. Las ASADAS no han recibido del AyA capacitación técnica o la ayuda administrativa y legal que requieren. Un participante lo detalló de la siguiente manera:

Nosotros le decimos al AyA que, si se supone que el AyA es la "madre" de las ASADAS, nosotros somos huérfanas... ellos no nos dan apoyo ni técnico ni administrativo ni legal... nunca nos capacitan en ningún tema... todo lo hacemos por nosotros mismos... peor aún, sentimos que ellos solo quieren hacernos desaparecer (ASADA N. 213, 20 de junio, 2014).

Las ASADAS han ayudado a las comunidades a organizarse para enfrentar mejor los problemas alrededor de las aguas subterráneas. También han impulsado a los pobladores a permanecer vigilantes de todo lo que sucede con las aguas subterráneas. Tal como destacó un entrevistado: "En esta comunidad, las ASADAS han sido muy importantes... en nuestras reuniones siempre discutimos cuáles son los nuevos desarrollos... qué quieren hacer... y si están haciendo las cosas apegados a la legalidad... estamos vigilantes...", (Organizaciones Comunales N.o 25, 22 de julio, 2013).

\section{El papel de las entidades gubernamentales y la respuesta de las comunidades}

Lo explicado anteriormente evidencia cómo el Gobierno y sus instituciones no han tomado en cuentas las condiciones climáticas ni las necesidades de las comunidades al decidir el modelo de desarrollo en la zona. Las instituciones públicas figuran, cada vez más, como objeto de denuncia y reacción, antes que como mediador (Estado de la Nación, 2009). Así las cosas, en las comunidades costeras de Santa Cruz ha crecido el descontento contra el sector privado a cargo de los negocios inmobiliarios y de turismo, y también contra las ineficientes entidades públicas. De hecho, los mismos representantes de instituciones gubernamentales admitieron las numerosas brechas en hacer cumplir las leyes ambientales. Tal como lo expresó un entrevistado del gobierno: "Aquí hay mucha falta de aplicación de la legislación ambiental... no podemos supervisar y controlar que todos estén usando sus concesiones de agua solo por la cantidad de agua concesionada... sabemos que también hay muchos pozos ilegales... pero no tenemos suficiente personal para venir y cerrar todos los pozos ilegales" (Entidad gubernamental local N. 22 , 21 de julio, 2013).

Debido a estas circunstancias, las comunidades costeras han sufrido una miríada de conflictos socioambientales a través de los años. Se estima que, en muchos de los conflictos socioambientales ligados al uso del agua ocurridos en Guanacaste, las instituciones públicas han sido directamente acusadas por negligencia 
(Estado de la Nación, 2009, 246). Quizás uno de los conflictos socioambientales más emblemáticos relacionados directamente con el acceso al agua ocurrió en 2002-2004 cuando un mega proyecto turístico quiso obtener una concesión de agua para extraer 180 litros por segundo del acuífero de Nimboyores. El mencionado acuífero es la principal reserva de agua potable para las comunidades costeras del cantón de Santa Cruz. El hotel que solicitaba la concesión ya tenía otra de 72 litros por segundo (CEDARENA, 2004), pero querían seguir expandiéndose.

Este acuífero se ubica a 15 kilómetros del hotel, lo que evidencia la escasez de agua en la región y la inversión que algunas compañías están dispuestas a hacer para garantizar a sus proyectos el acceso al agua. En aquel entonces (2002), ya el hotel tenía una concesión de agua del acuífero de Huacas-Tamarindo, del cual obtenía una importante cantidad de agua. En aquel entonces, las comunidades se dieron cuenta de lo que ocurría porque el hotel comenzó a construir la cañería para transportar el agua subterránea. Esa cañería atravesaba algunas de las comunidades y, así, sus pobladores notaron lo que estaba sucediendo. Comenzó entonces la organización comunal para hacer protestas y cierre de vías, con el fin de detener la construcción de la cañería. Al mismo tiempo, los vecinos acudieron a las instituciones públicas para averiguar en qué etapa iba el proceso de la concesión y presentar sus reclamos, en donde argumentaban que las comunidades no habían sido informadas y que el otorgamiento de esta concesión al hotel afectaría el acceso al agua de muchas personas. En resumen, y de boca de un participante: "Estábamos realmente preocupados, era un asunto enorme... pensábamos que, si le daban la concesión al hotel... no íbamos a tener agua" (Organización Comunal N. 26, 22 de julio, 2013).

Al final, la concesión no se le otorgó al hotel, principalmente debido a los reclamos de los pobladores que indicaban que se pondría en riesgo el acceso al agua de muchas personas. Además, algunas instituciones estatales como por ejemplo SENARA determinaron que el proyecto debía ser evaluado ambientalmente, hecho que no había sucedido. De acuerdo con los estudios recientes de SENARA (institución estatal a cargo de elaborar estudios hidrológicos explicado en la sección 3), el acuífero de HuacasTamarindo está sobreexplotado y en peligro de contaminación por intrusión de agua salina (SENARA, 2015).

Desde entonces, se han presentado muchos otros conflictos socioambientales en estas comunidades y tensión entre la población, el sector privado y las entidades gubernamentales. Por eso, los y las vecinas han estado organizados y vigilantes con lo que acontece en su zona. Al final, el conflicto constituyó una oportunidad para discutir el modelo de desarrollo que quieren para la región, la necesidad de un plan de manejo integral de los recursos naturales que permita proteger las aguas subterráneas y garantizar a las comunidades su acceso. Pese a esto, las entidades gubernamentales y el sector privado tienen una idea muy diferente del tipo de desarrollo que quieren para la región (Estado de la Nación, 2009). 
Tal como declararon la mayoría de los y las entrevistadas de estas comunidades, las instituciones públicas, tales como la municipalidad de Santa Cruz y el gobierno central (por ejemplo el Instituto Costarricense de Turismo (ICT) y el AyA), continúan con la promoción del turismo masivo y el negocio inmobiliario, mientras que las comunidades quisieran dirigirse a un turismo comunitario y ecológico. En resumen, una participante mencionó lo siguiente: "lo que necesitamos aquí en Guanacaste es un turismo más sostenible... más rural... donde las comunidades seamos los actores principales... no como se ha dado hasta el momento"' (Asada N. ${ }^{\circ} 27,22$ de julio, 2013).

Al igual que el caso de estudio de Guácimo, para comprender mejor las causas por las cuales se pudo manejar, hasta cierto punto, el conflicto socioambiental, es vital analizar algunos elementos existentes en las comunidades costeras que les permitieron organizarse y mejorar algunos de los problemas existentes. Un elemento que destaca en este caso particular y que ayudó a la organización fue que las comunidades se dieron cuenta de que había una crisis hídrica. Estos pueblos han tenido, y tienen en la actualidad, serios problemas de disponibilidad de agua, en especial durante la estación seca, debido a la ausencia de precipitaciones (Kuzdas 2014). Esta circunstancia en conjunto con otros factores tales como los discutidos anteriormente (crecimiento del turismo y desarrollo inmobiliario sin tomar en cuentas las condiciones climáticas de la zona, falta de una adecuada planificación, y limitada capacitación de las ASADAS) han agravado la crisis hídrica que sufre esta zona. Tal como lo argumentó un entrevistado: "Hay muchos problemas en esta región... pero, con seguridad se puede decir que, el que afecta a más gente es la falta de agua... especialmente durante la estación seca... sin embargo, cada vez hay más hoteles y desarrollos inmobiliarios" (Asada N. ${ }^{\circ}$ 28, 23 de julio, 2013).

Otra condición que permitió que las comunidades se organizaran y solventaran ciertos problemas existentes en este caso de estudio, fue el liderazgo comunal. Los pueblos costeros tienen líderes que han convencido a muchos de participar en la gobernanza de aguas subterráneas, entre los que destacan las ASADAS y las asociaciones de desarrollo comunal. Las ASADAS, por ejemplo, se han reunido con gente de las comunidades para hablarles de la importancia del agua, de los problemas existentes, de cómo podrían contribuir a mejorar los problemas urgentes a través del trabajo conjunto. Tal como lo expresó un participante:

Tenemos que proteger nuestras playas, nuestros bosques y nuestra agua para las futuras generaciones...y sabemos que no podemos contar con ninguno de los organismos gubernamentales, porque no les importamos... a ellos solo les importa el turismo, el negocio inmobiliario y todo lo que les dé dinero (ASADA N. 213, 20 de junio 2014).

Al igual que en Guácimo, otra condición que facilitó la organización de los habitantes de estas comunidades y de las ASADAS fue que las comunidades 
son pequeñas y considerablemente homogéneas. La población total de estos pueblos ronda las 35000 personas. Tal como explicó un entrevistado:

Estos poblados son muy pequeños, todos nos conocemos. A pesar de que, debido al turismo y al crecimiento inmobiliario el lugar está creciendo, muchos no viven aquí...incluso quienes tienen una casa.... solo vienen en vacaciones... pero, nosotros estamos siempre... y nos importa la comunidad...la mayoría tenemos principios y valores similares (Organización comunal N. ${ }^{\circ} 219,22$ de junio 2014).

Dicha condición ha ayudado a los pueblos costeros a trabajar juntos y a desarrollar un sentido de pertenencia.

En resumen, este caso de estudio revela algunas razones por las cuales emergen los conflictos socioambientales como, por ejemplo, la falta de capacidad o negligencia por parte de las instituciones públicas en los ámbitos central y local y algunas condiciones que ayudan a sobrellevarlos, como el liderazgo comunal. Las comunidades costeras de Santa Cruz continúan enfrentando el problema de un modelo de desarrollo inadecuado ya que no toma en cuenta las condiciones climáticas ni las necesidades de sus pobladores. Sin embargo, se ha dado algún progreso, como mayor organización y control de parte de las ASADAS y de los poblados. Las ASADAS y las comunidades continúan alerta $\mathrm{y}$, en este momento, estudian los impactos de la propuesta estatal para construir un acueducto regional que si bien es cierto traería agua para las comunidades sigue promoviendo la idea del mismo tiempo de desarrollo. Tal como lo indicó un participante: "No hemos visto la evaluación de impacto ambiental, o el estudio de viabilidad... no sabemos con certeza cuáles serían los impactos de explotar el acuífero de Nimboyores... por eso, no estamos de acuerdo con este proyecto" (ASADA N. ${ }^{\circ}$ 214, 20 de junio, 2014).

\section{Conclusión}

Este artículo muestra varios elementos que ayudan a comprender en qué medida los conflictos socioambientales pueden evitarse o manejarse y alcanzar una efectiva gobernanza de las aguas subterráneas. Con base en experiencias de campo, se ha proporcionado información para contestar esta interrogante. Es evidente que una medida fundamental para evitar conflictos socioambientales es que las entidades gubernamentales funcionen como garante de la implementación de la legislación ambiental. Por eso, cuando se ha violentado dicha legislación ha habido una proliferación de conflictos de este tipo. La negligencia por parte de entidades estatales a nivel central y local permiten al sector privado violar fácilmente la legislación ambiental, lo cual, como consecuencia, provoca daños al ambiente. Por ejemplo, permiten la contaminación de las aguas subterráneas que afecta severamente a las comunidades más vulnerables. También este artículo muestra que existe una evidente contradicción 
entre la política ambiental (de conservación y de país verde con miras a convertirse en carbono neutral) y la política económica (de monocultivos expansivos, turismo masivo e inmobiliarias en zonas con escasez de agua) de Costa Rica.

Los dos casos de estudio discutidos en este artículo presentaban el mismo problema, específicamente, la falta de implementación de legislación ambiental por parte de las autoridades gubernamentales, lo cual afecta directamente al ambiente y a las comunidades aledañas. Por ello, en cierta medida, se obtiene la misma respuesta de la gente: una organización comunal que coordina denuncias legales y protestas. También, los dos casos de estudio evidencian la falta de un modelo de desarrollo integral planificado y sustentable por parte de las entidades gubernamentales y de acuerdo con la política ambiental del país. El caso de estudio de Guácimo mostró cómo la actividad económica de las plantaciones piñeras contamina el agua debido a la falta de vigilancia y control de dicha actividad por parte de las autoridades responsables. Por ejemplo, no se aplicaba la prohibición de instalar plantaciones piñeras a la par de los manantiales y en áreas de recarga acuífera. En este caso de estudio, además se evidencia que el Gobierno no ha proporcionado más opciones para el desarrollo de estas comunidades que la existencia de monocultivos expansivos como la piña.

En las comunidades costeras, los principales problemas han sido la sobreexplotación del recurso hídrico y la intrusión salina, los cuales han sido causados en gran medida por actividades económicas tales como el turismo masivo. Este caso de estudio, de nuevo, ilustró que los problemas se derivan en gran medida por la falta de aplicación de la legislación ambiental y el ineficiente control que dichas actividades tienen por parte de instituciones públicas. Este caso de estudio también ilustró que el tipo de turismo y negocio inmobiliario, que impulsa el Estado, no contempla que Santa Cruz es una zona vulnerable y seca y que el área no tiene un desarrollo acorde con sus condiciones biofísicas (Kuzdas, 2014).

En el caso de Guácimo, el papel de la municipalidad ha sido muy importante para evitar más conflictos socioambientales y una mejor gobernanza de aguas subterráneas. La municipalidad ha impulsado importantes regulaciones para el cantón, que pretenden evitar que las plantaciones agrícolas se instalen en áreas vulnerables como acuíferos y zonas de recarga. Por ejemplo, la municipalidad de Guácimo implementó por cinco años una moratoria a la expansión de la actividad piñera. Sin embargo, como se discutió en este artículo, la Sala Constitucional considera que los derechos del comercio y la propiedad privada son más importantes que las medidas de precaución establecidas por la municipalidad de Guácimo para proteger las aguas subterráneas. A pesar de que la moratoria ya no está vigente, hubo algunos logros, como una municipalidad más consolidada y vigilante. Este caso ilustra que, a pesar de que la municipalidad tomó una buena decisión como respuesta a las presiones populares para evitar más daño a las aguas subterráneas, el gobierno central no 
sintió esa presión. Además, muestra que la Sala Constitucional estaba más preocupada por la afectación económica al desarrollo de plantaciones piñeras que por tener una gobernanza sustentable de aguas subterráneas y comunidades sin agua contaminada.

En el caso de estudio de las comunidades costeras de Santa Cruz, fue significativo que el gobierno no otorgara una gran concesión a un mega proyecto turístico, lo cual hubiera ocasionado escasez de agua a mucha gente. Además, fue importante que el SENARA dijera que, antes de dar tal tipo de concesión, es importante elaborar una evaluación ambiental integral del proyecto en su totalidad. Este artículo evidencia que a veces son necesarias las protestas y las denuncias legales por parte de las comunidades, con el fin de ejercer presión pública a las instituciones, de manera que se involucren y sean vigilantes, ya que fue hasta después de que las comunidades organizaron manifestaciones, protestas y denuncias legales que se decidió que la concesión no se iba a otorgar.

Otro punto destacable para estas comunidades costeras es la propuesta gubernamental de desarrollar un acueducto regional, con el fin de proveer agua para su uso actual y futuro, lo que incluye el uso del acuífero de Nimboyores. El conflicto socioambiental discutido en este artículo ocurrió porque el gobierno estaba a punto de otorgar una gran concesión de agua de este acuífero a un hotel. Por lo tanto, si el gobierno sigue adelante con esta propuesta de utilizar el acuífero de Nimboyores, es muy importante que el proceso de toma de decisión incluya el apoyo de las comunidades que se puedan ver potencialmente afectadas.

Los dos casos de estudio demuestran la importancia de consolidar la conciencia ambiental (entendimiento del impacto que provocan las actividades humanas en el entorno) y capacitar a los y las funcionarias de las entidades gubernamentales acerca del impacto que las diversas actividades económicas tienen sobre los recursos naturales y las comunidades aledañas. Además, es fundamental crear más mecanismos de participación en procesos de toma de decisión pues, tal como lo han señalado diversos autores, eso tiende a ayudar a garantizar una gobernanza eficiente (Varady et al., 2013, Akhmouch y Clavreul, 2016). La gobernanza de las aguas subterráneas requiere participación activa e involucramiento de todas las partes interesadas para ayudar a asegurar una creciente rendición de cuentas (Kemper 152). En definitiva, como ha subrayado Varady et al., la participación en la gobernanza de las aguas subterráneas se justifica en términos de sustentabilidad y empoderamiento. Por eso, parece que la participación es fundamental para asegurar que sean bien administradas y que se tome en cuenta las necesidades de la mayoría de la gente.

Finalmente, sería valioso para ambos casos de estudio fomentar las redes entre organizaciones comunales y compartir las experiencias de este tipo, con sus logros y retos. En este sentido, las organizaciones comunales podrían percatarse que no están solas y que no son las únicas que sufren este tipo de problemas y además podrían compartir sus experiencias con miras a fortalecer sus acciones (Cuadrado-Quesada, 2014). 
Finalmente, es importante mencionar que, mientras se llevaba a cabo el trabajo empírico de esta investigación (2012-2015) se dio un cambio de gobierno en Costa Rica. ${ }^{8}$ Muchos de los entrevistados se mostraron a favor de dicho cambio y sugirieron que, probablemente, se iban a dar algunos cambios positivos. Es necesario realizar un seguimiento a esta investigación para conocer si, efectivamente, con el nuevo gobierno la situación ha mejorado.

\section{Notas}

$1 \quad$ Este artículo fue originalmente escrito en inglés y presentado en la 8. a Nordic Latin American Research Network Conference, llevada a cabo del 11 al 13 de junio del 2015 en Helsinki, Finlandia. La versión en español fue traducida y editada por Yazmín Montoya Jiménez.

2 Para más información sobre Adaptive Theory, ver Layder, Sociological Practice: Linking Theory and Social Research (SAGE, 1998).

3 Para más información sobre el derecho humano de acceso al agua, se recomienda ver los votos 00-02755, del 24 de marzo de 2000; 2002-10776, del 14 de noviembre de 2002, y 4654-2003, del 27 de mayo de 2003 de la Sala Constitucional de la Corte Suprema de Justicia de Costa Rica.

El riego es el principal uso de las aguas subterráneas. Sin embargo, este documento no discute propiamente las implicaciones de este empleo.

Para más información sobre la Matriz del Agua, ver Arias, 'Vulnerabilidad y Protección del Agua Subterráneas: Valor de la Matriz del Uso del Suelo de SENARA' (2012) 228 Ambientico 9-13.

Para más información ver el Voto de la Sala Constitucional No 2009-00262.

Por ejemplo, Siquirres, Pococí, Upala, Los Chiles, Guatuso, Buenos Aires, entre otros (Cuadrado-Quesada y Castro-Vargas, 2009).

\section{Bibliografía}

Aguirre D y Arboleda E, 'Impacto Ambiental del Cultivo de Piña y Características de Este (Caso Siquirres)' (2008) 177 Revista Ambiental Ambientico 3-8.

Akhmouch, Aziza y Delphine Clavreul. 'Stakeholder Engagement for Inclusive Water Governance: "Practicing What We Preach" with the OECD Water Governance Initiative' 8, 204 (2016): 1-17.

Arias, Mario E. “Gestión del Recurso Hídrico y Uso del Agua”. Programa Estado de la Nación en Desarrollo Humano Sostenible/Programa Estado de la Nación, Costa Rica. 2009.

Astorga, Yamileth. “La Gestión de Aguas Subterráneas: Un Espacio de Conflicto”. Programa Estado de la Nación en Desarrollo Humano Sostenible /Programa Estado de la Nación, Costa Rica. 2009. 
Ballestero-Salaverry, Andrea, 'Introducción' en Aportes para la Discusión sobre el Derecho Humano de Acceso al Agua, Ballestero-Salaverry, Andrea (Ed.), Centro de Derecho Ambiental y de los Recursos Naturales (CEDARENA) 2009.

Boulton, Andrew J., et al. "Biodiversity, Functional Roles and Ecosystem Services of Groundwater Invertebrates". Invertebrates Systematics, 22, 2 (2008): 103-116.

Castro-Cordoba, Rolando, et al. “Gestión Local y Participativa del Recurso Hídrico en Costa Rica". Centro de Derecho Ambiental y de los Recursos Naturales (CEDARENA). 2004.

Coleman, Thomas. "Who Owns the Water? An Analysis of Water Conflicts in Latin American and Modern Water Law". Intersections 12, 2 (2012): 1-19.

Cuadrado-Quesada, Gabriela. “Legalización de la Contaminación de las Agua para Consumo Humano (caso del Diurón y el Bromacil)”. Ambietico 177 (2008): 11-12.

Cuadrado-Quesada, Gabriela. "Groundwater Governance and Spatial Planning Challenges, Examining Sustainability and Participation on the Ground". Water International 39, 6 (2014): 798-812.

Cuadrado-Quesada, Gabriela y Soledad Castro-Vargas. La expansión del monocultivo de la piña en detrimento de los derechos humanos. México D.F.: Red sugar, green deserts. HIC-AL y FIAN (2009).

De Albuquerque, Catarina. Mission to Costa Rica. Geneva: Human Rights Council. 2009.

Dobbin, Kristin B. y Brinda Sarathy. "Solving Rural Water Exclusion: Challenges and Limits to Co-Management in Costa Rica". Society \& Natural Resources 28, 4 (2015): 388-404.

Fresh Action Network-Central America (FANCA). "Water Boards in Central America. Assessment of Local Management of Water Resources. A Comparative Study" (2006).

Foster, Stephen y Héctor Garduño. "Groundwater Resource Governance, Are Governments and Stakeholders Responding to the Challenge?". Hydrogeology Journal, 21, 2(2013): 317-320.

Guzmán-Arias, Isabel y Julio C. Calvo-Alvarado. “Planning and Development of Costa Rica Water Resources: Current Status and Perspectives". Tecnología en Marcha, 26, 4 (2003): 52-63.

Instituto Nacional de Estadística y Censos (INEC). "Proyección de Población al 30 de junio de 2016".

Kruse, Thomas. "La Guerra del Agua en Cochabamba Bolivia: Terrenos Complejos, Convergencias Nueva". Sindicatos y Nuevos Movimientos Sociales en América Latina. Comp. Enrique De la Garza. 2005.121-161.

Kuzdas, Chirstopher, et al. "Environmental Management, Sustainability Appraisal of Water Governance Regimes: The Case of Guanacaste, Costa Rica". Environmental Management, 54, 2 (2014): 205-222.

Liamputtong, Pranee y Douglas Ezzy. “Qualitative Research Methods". EE. UU.: Oxford University Press, 2005.

Monge Esteban, Paz Luisa y Ovares Carolina, Transparencia y Rendición de Cuentas en las ASADAS, Manual para las Asociaciones Administradoras de Sistemas de Acueductos y Alcantarillados Sanitarios (ASADAS) de Costa Rica, Centro de Derecho Ambiental y de los Recursos Naturales (CEDARENA) 2013.

Morgan, Browen. “Comparative Regulatory Regimes in Water Service Delivery: Emerging Contours of Global Water Welfarism?". Comparative Research in Law E Political Economy. Research Paper, 4, 7(2008): 1-38.

Morris Brian L, et al. "Groundwater and its Susceptibility to Degradation: A Global Assessment of the Problem and Options for Management" (UNEP 2003). 
Programa Estado de la Nación (Costa Rica). “Decimoquinto Primer Informe Estado de la

Nación en Desarrollo Humano Sostenible". Programa Estado de la Nación, 2009.

Programa de las Naciones Unidas para el Desarrollo (PNUD). "Atlas del Desarrollo Humano Cantonal de Costa Rica". Universidad de Costa Rica, 2011.

Programa Estado de la Nación (Costa Rica). “Vigésimo Primer Informe Estado de la Nación en Desarrollo Humano Sostenible". Programa Estado de la Nación, 2015.

Ruepert, Clemens et al., "Vulnerabilidad de las Aguas Subterráneas a la Contaminación por Plaguicidas en Costa Rica", Instituto Regional de Estudios en Toxicología (IRET), Universidad Nacional, Costa Rica (2005).

Shah Tushaar, "Taming the Anarchy, Groundwater Governance in South Asia", Resources for the Future (RFF), (2009).

Varady, Robert, et al. "Groundwater Policy \& Governance". Thematic Paper No 5, Groundwater Governance: A Global Framework for Country Action, Global Environmental Found (GEF), 2013, Rome.

Wijnen, Marcus, et al. "Managing the Invisible. Understanding and Improving Groundwater Governance". World Bank, 2012, Washington, DC.

World Water Assessment Programme (WWAP). The United Nations World Water Development Report 4, Managing Water under Uncertainty and Risk, (UNESCO) 2012, Paris.

Yin, Robert. "Case Study Research: Design and Methods". SAGE Publications, 2014, Thousand Oaks, CA.

Zartman, William. “Comparative Case Studies". International Negotiation, 10, 1 (2005): 3-16.

\section{Legislación Costarricense}

Ley de Aguas Ley No 276, 1942.

Código Penal, Ley No. 4573, 1970.

Ley General de la Salud, Ley No. 5385, 1973.

Ley Orgánica del Ambiente, Ley No. 7554, 1995.

Ley Forestal, Ley No. 7575, 1996.

Ley de Biodiversidad, Ley No. 7788, 1998.

\section{Resoluciones Sala Constitucional de la Corte Suprema de Justicia, Costa Rica}

Sala Constitucional de la Corte Suprema de Justicia, Resolución N. 200401923, San José, catorce horas cincuenta y cinco minutos del veinticinco de febrero de dos mil cuatro.

Sala Constitucional de la Corte Suprema de Justicia, Resolución No. 20130013939, San José, once horas treinta minutos del dieciocho de octubre de dos mil trece. 


\section{Resoluciones Municipales, Costa Rica}

Concejo Municipal de Guácimo, Acta No. 34-2007, Sesión Ordinaria No. 272007, celebrada por el Concejo Municipal de Guácimo, el 3 de Julio del 2007. Publicada en la Gaceta No. 126 del 1 de Julio del 2008.

\section{Resoluciones Fiscalía Ambiental Adjunta, Costa Rica}

Fiscalía Ambiental Adjunta Resolución No. 24536-2013.

\section{Informes de instituciones públicas, Costa Rica}

Servicio Nacional de Aguas Subterráneas, Riego y Avenamiento (SENARA), Evaluación del Potencial y Demanda Hídrica Subterránea en el Acuífero Costero Huacas - Tamarindo, Santa Cruz, Guanacaste, Costa Rica. Mayo 2003.

Servicio Nacional de Aguas Subterráneas, Riego y Avenamiento (SENARA), Monitoreo de los Acuíferos 'Huacas-Tamarindo y Potrero-Caimital' 30 de enero del 2015. Realizado por la Geóloga Clara Agudelo y el Ingeniero Carlos Romero.

Gabriela Cuadrado Quesada. Costarricense, abogada, graduada de la Facultad de Derecho de la Universidad de Costa Rica. Obtuvo una maestría en Derechos Humanos y Educación para la Paz en la Universidad Nacional, Costa Rica. Posteriormente adquirió otra maestría en Water and Coastal Management y Environmental and Infraestructure Planning otorgada por las universidades de Oldenburg (Alemania) y Groningen (Países Bajos). En el 2017 obtuvo su doctorado en Derecho por la Universidad de New South Wales, Australia. Actualmente se desempeña como investigadora en UNESCO-IHE, Instituto para la Educación del Agua en Delft (Países Bajos). Algunas de sus publicaciones incluyen "Groundwater governance and spatial planning challenges: examining sustainability and participation on the ground" (2014), "People's Tribunals: a progressive mechanism to achieve justice" (2014), "El reconocimiento del derecho a un medio ambiente sano en el derecho internacional y en Costa Rica" (2009) y "Legalización de la contaminación de aguas para consumo humano (caso del Diurón y el Bromacil)" (2008).

Contacto: g.quesada@un-ihe.org

ORCID: 0000-0002-0271-1522 